\title{
Identification and Quantification of Alkaloid in KHR98 and Fragmentation Pathways in HPLC-Q-TOF-MS
}

\author{
Jiakun Long, Yang Wang, Chen Xu, Tingting Liu, Gengli Duan,**\# and Yingjia Yu**\# \\ Department of Pharmaceutical Analysis, School of Pharmacy, Fudan University; No. 826 Zhangheng Road, Shanghai \\ 201203, China. \\ Received September 3, 2017; accepted January 17, 2018; advance publication released online March 2, 2018
}

\begin{abstract}
Uncaria rhynchophylla is woody climber plant distributed mainly in China and Japan, the stems and hooks of which can be collected as "Gou-Teng" for the treatment of hyperpyrexia, epilepsy and preeclampsia. Fudan University first manufactured KHR98, the extract of Uncaria rhynchophylla. In order to study the active components and structural information of KHR98, we established a HPLC coupled with quadrupole time-of-flight (Q-TOF)-MS method for rapid analysis of alkaloids. In qualitative analysis, a total of eight compounds, including four known alkaloids and four unknown components, were detected and identified. The fragmentation behaviors, such as the fragment ion information and the fragmentation pathways of the eight components were summarized simultaneously, and the concentration of the above components was determined by HPLC-MS method. The quantitative method was proved to be reproducible, precise and accurate. This study shed light on the standardization and quality control of the KHR98 and provided a foundation for the further research on pharmacology, follow-up clinical research and New Drug Applications.
\end{abstract}

Key words Uncaria rhynchophylla (UR); alkaloid; fragmentation pathway; identification; qualification; HPLC coupled with quadrupole time-of-flight (Q-TOF)-MS

Uncaria rhynchophylla (UR) is mainly distributed in China and Japan, ${ }^{1)}$ and is generally used to treat ailments in cardiovascular and central nervous systems, such as dizziness, convulsions, cerebral arteriosclerosis and hypertension, etc. ${ }^{2-5)}$ The phytochemical studies of UR have resulted in the discovery of various types of compounds, including indole alkaloids, triterpenes, flavonoids and phenylpropanoids, ${ }^{6)}$ of which the indole alkaloids, for instance, rhynchophylline, isorhynchophylline, corynoxeine, isocorynoxeine and hirsutinea, are commonly recognized as important bioactive ingredients closely related to the pharmacological activities. ${ }^{7,8)}$ These alkaloids exhibit a number of pharmacological effects, such as decreased blood pressure, vasodilatation, sedation and protection against ischemia-induced neuronal damage. ${ }^{9,10)}$ So it is important to separate and analyze those alkaloids. Up till now, multiple researches have been made in this area and several composition analysis methods have been employed. HPLC-UV technique has been adopted in previous researches on alkaloids, such as the study on the screening and identification of six oxindole alkaloids and four indole alkaloids in Uncaria rhynchophylla by HPLC. ${ }^{11)}$ Apart from that, the HPLC-MS technique, which is able to provide both analytical separation and structural determination of unknown bioactive compounds, has previously been proposed as a technique of choice for the analysis of these alkaloids from Uncaria rhynchophylla ${ }^{12,13)}$; for instance, ten oxindole alkaloids and four glycosidic indole alkaloids have been identified using LCatmospheric pressure chemical ionization (APCI)-MS method, ${ }^{12)}$ and a total of 29 compounds, comprising 18 alkaloids, 6 flavonoids and 5 quinic acids have been identified employing HPLC-diode array detector (DAD)-quadrupole time-of-flight (Q-TOF)-MS method ${ }^{1)}$ in previous studies.

\footnotetext{
\# These authors contributed equally to this work.

KHR98, a dark brown powder, is the extract of a traditional Chinese medicine Uncaria rhynchophylla. It was first produced by Fudan University and belonged to the State Category $\mathrm{V}$ New Drug. According to the requirements of State Food and Drug Administration (SFDA) for the registration and classification of traditional Chinese medicine and natural medicine ${ }^{14)}$ the effective constituents should account for more than $50 \%$ of the extracts for the State Category V New Drug. But for KHR98, the alkaloids contained in the effective constituent are not clear. In this study, four kinds of alkaloid components, namely rhynchophylline, isorhynchophylline, corynoxeine and isocorynoxeine have been detected to contain KHR98. Then, the possible fragmentation pathways of the four kinds of alkaloids were inferred from the accurate molecular weight and fragment information provided by the high resolution mass spectrometer (Q-TOF). Next, the structural information and fragmentation pathways of four unknown components (hirsutine, dihydrocorynantheine, corynantheine, hirsuteine or theirs isomers) were interpreted in KHR98 through higher quality structural information offered by the accurate mass measurement of all the protonated molecules and diagnostic fragment ions. Last, the above components were quantified using HPLC-MS method.

Four known indole alkaloids were detected and four unknown components were identified for KHR98 as a whole. HPLC-MS/MS was used to identify diagnostic fragment ions and interpret fragmentation pathways of known and unknown alkaloids of KHR98. A precise and sensitive HPLC-MS method was validated to simultaneously determine these substances. Therefore this study can be a foundation for further pharmacological study, clinical researches and New Drug Application of this State Category V New Drug, and a reference for future work. 
Table 1. Targeted MS-MS Table

\begin{tabular}{lccccc}
\hline \hline Alkaloid & Prec. $m / z$ & Ret. time $(\mathrm{min})$ & Delta ret. time $(\mathrm{min})$ & Iso. width & Collision energy \\
\hline Corynoxeine & 383.1975 & 15.059 & 0.5 & Medium $(\sim 4 m / z)$ & Medium $(\sim 4 m / z)$ \\
Isocorynoxeine & 383.1975 & 19.175 & 0.5 & Medium $(\sim 4 m / z)$ \\
Isorhynchophylline & 385.2128 & 14.514 & 0.5 & Medium $(\sim 4 m / z)$ & 25 \\
Rhynchophylline & 385.2128 & 19.370 & 0.5 & Medium $(\sim 4 m / z)$ & 30 \\
Corynantheine & 367.2024 & 17.422 & 0.5 & Medium $(\sim 4 m / z)$ & 30 \\
Hirsuteine & 367.2024 & 17.984 & 0.5 & Medium $(\sim 4 m / z)$ & 30 \\
Hirsutine & 369.2177 & 17.431 & 0.5 & Medium $(\sim 4 m / z)$ \\
Dihydrocorynantheine & 369.2177 & 19.115 & 0.5 & & 30 \\
\hline
\end{tabular}

\section{Experimental}

Chemicals and Drugs The Uncaria rhynchophylla extract, KHR98 (lot number: 43, 44, 45), was kindly provided by Fudan University (FU, Shanghai, China). Standard compounds, including corynoxeine, isocorynoxeine, rhynchophylline, hirsutine and isorhynchophylline were purchased from Seebio Biotech (Shanghai) Co., Ltd. (Shanghai, China). The purity of all the standard compounds was over 98\%. Ammonium acetate, analytical grade, was purchased from Sinpharm Chemical Reagent Co., Ltd. (Shanghai, China). Acetonitrile, HPLC grade, was purchased from Merck (Darmstadt, Germany). Deionized water used for LC-MS/MS and LC-MS was freshly processed through the Milli-Q plus system (Millipore, U.S.A.).

Instrumentation The above alkaloids were carried out by an Agilent 1290 Infinity Series HPLC System (Agilent, Palo Alto, CA, U.S.A.) which was equipped with a pump, an automatic sampler, a column oven and an automatic degasser. A 6538 Accurate-Mass Q-TOF-MS device (Agilent Technologies, CA, U.S.A.) interfaced with an electrospray ionization (ESI) source was employed for the HPLC-MS/MS analyses in positive ionization mode. The raw data acquisition and processing were performed using Analyst software Version 1.7.

HPLC-MS and HPLC-MS/MS Conditions Samples were separated on a Xtimate $\mathrm{C}_{18}$ column $(250 \times 4.6 \mathrm{~mm}$ i.d., $5 \mu \mathrm{m}$; Welch ${ }^{\circledR}$, China). The mobile phase consisted of $10 \mathrm{~mm}$ Ammonium acetate solution and acetonitrile at 55:45 (v/v) delivered with a flow rate of $0.8 \mathrm{~mL} / \mathrm{min}$. The injection volume was $2 \mu \mathrm{L}$ and the column temperature was maintained at $30^{\circ} \mathrm{C}$. The divergence ratio was $3: 1$.

The mass spectrometer was equipped with an ESI source operating in positive ion mode. The following operating parameters were used: drying gas (N2) flow rate, $12 \mathrm{~L} / \mathrm{min}$ at $300^{\circ} \mathrm{C}$; capillary voltage, $3500 \mathrm{~V}$; atomized pressure, $45 \mathrm{psig}$; fragmentor voltage, $175 \mathrm{~V}$; Skimmer voltage, $65 \mathrm{~V}$; OCR $1 \mathrm{RF}$ Vpp $750 \mathrm{~V}$; mass range, $m / z$ 100-1000. The exact molecular weight was measured using a ten point correction method $(\mathrm{m} / \mathrm{z} 112.9856,301.9981,601.9790,1033.9881$, 1333.9689, $1633.9498,1933.9306,2233.9115,2533.8923,2833.8731)$. Other mass parameters are shown in Table 1.

Preparation of Standard and Sample Solutions for Qualitative Analysis

\section{Standard Solutions}

Stock solutions $(500 \mu \mathrm{g} / \mathrm{mL})$ of four standard substances (corynoxeine, isocorynoxeine, rhynchophylline and isorhynchophylline) were prepared in methanol. Each stock solutions were transferred to a volumetric flask and mixed to make a standard solution with the concentration of $5 \mu \mathrm{g} / \mathrm{mL}$.

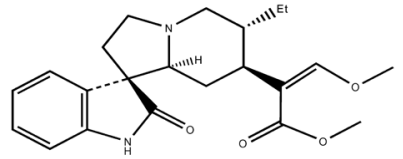

(A) Rhynchophylline

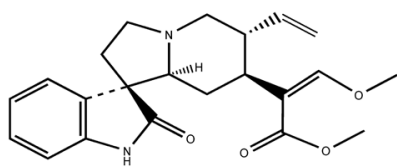

(C) Corynoxeine

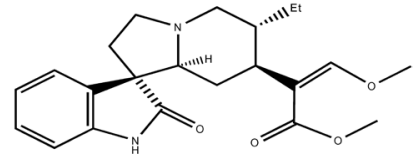

(B) Is orhynchophylline

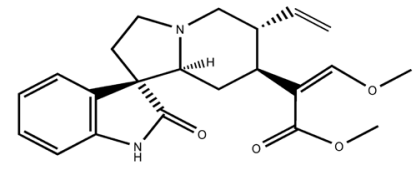

(D) Isocorynoxeine
Fig. 1. Structures of (A) Rhynchophylline, (B) Isorhynchophylline, (C) Corynoxeine and (D) Isocorynoxeine

\section{Sample Solutions}

The stock solutions of KHR98 was $1 \mathrm{mg} / \mathrm{mL}$ in methanol. The KHR98 sample solutions were prepared by diluting the KHR98 stock solutions with methanol to the concentration of $50 \mu \mathrm{g} / \mathrm{mL}$.

Preparation of Standard and Sample Solutions for Quantitative Analysis

Standard Solutions

Samples for five standard substances (corynoxeine, isocorynoxeine, rhynchophylline, hirsutine and isorhynchophylline) were accurately weighed and dissolved in methanol to make mixed stock solutions at $10 \mu \mathrm{g} / \mathrm{mL}$. The standard solutions were prepared with the appropriate dilution of stock solutions with methanol.

Sample Solutions

This method was in accord with the method of making sample solutions for qualitative analysis.

Linearity

The standard curves were established by plotting peak area ratio of alkaloid over concentration, ranging from 1 to $10 \mu \mathrm{g} / \mathrm{mL}$ ( $x$-axis). The stock solutions containing corynoxeine, isocorynoxeine, rhynchophylline, isorhynchophylline and hirsutine were diluted to concentrations of 1, 3, 5, 7 and $10 \mu \mathrm{g} / \mathrm{mL}$.

Limit of Detection (LOD) and Limit of Quantitation (LOQ)

The stock solutions containing five standard substances were diluted to concentrations ranging from $0.01 \mu \mathrm{g} / \mathrm{mL}$ to $1 \mu \mathrm{g} / \mathrm{mL}$. Under the present chromatographic conditions, the limits of LOD and LOQ were determined at a signal-to-noise ratio $(S / N)$ of 3 and 10 , respectively.

Precision

Took the standard solutions with the concentrations of 1 , 5 and $10 \mu \mathrm{g} / \mathrm{mL}$ to analysis under corresponding chromato- 


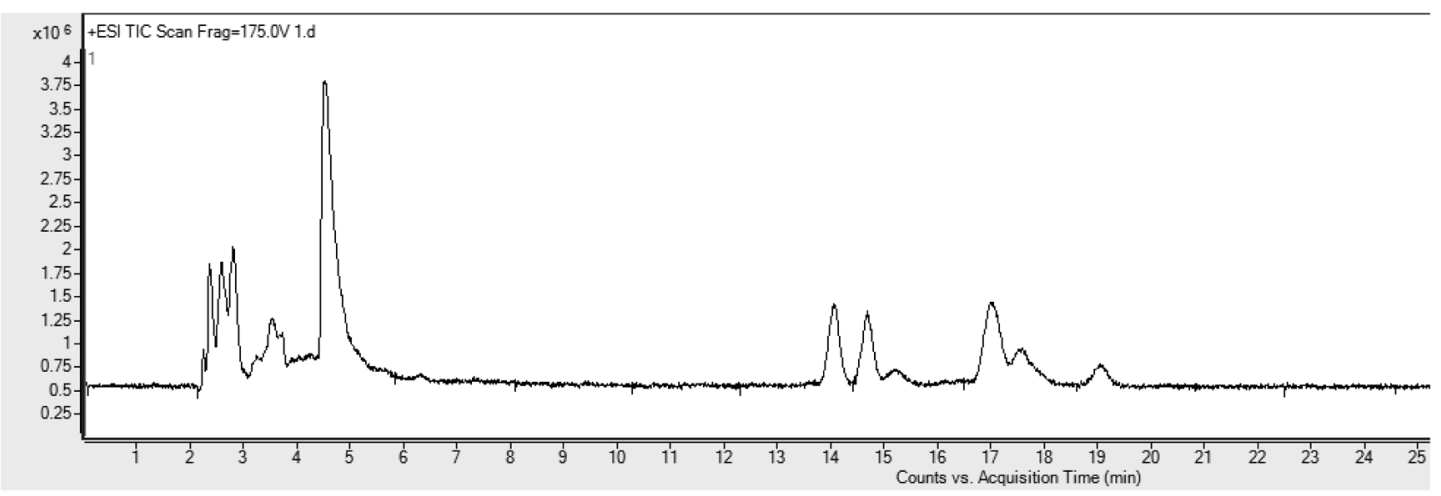

Fig. 2. TIC Chromatography

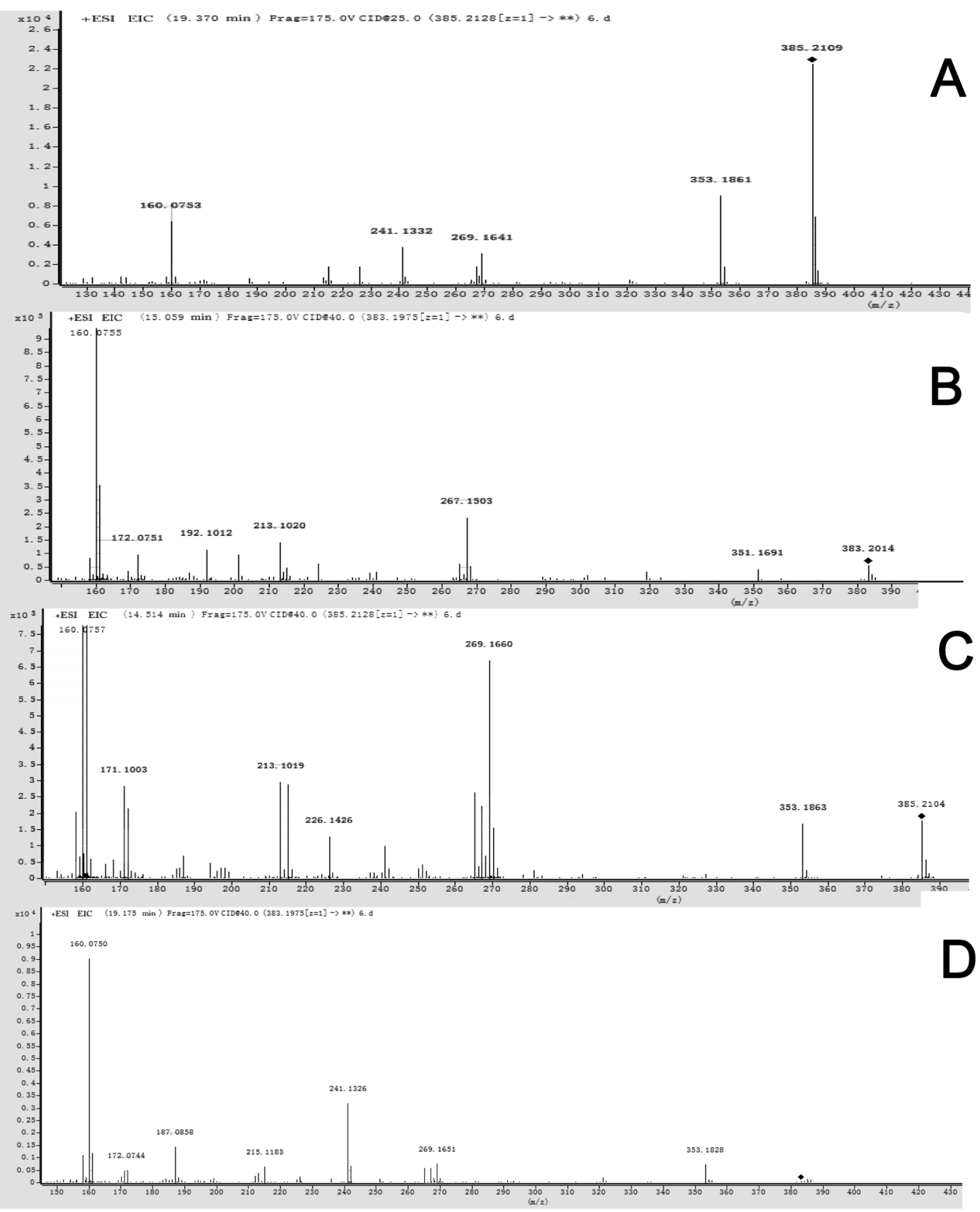

Fig. 3. MS/MS Spectra of (A) Rhynchophylline, (B) Isorhynchophylline, (C) Corynoxeine and (D) Isocorynoxeine 
(1)

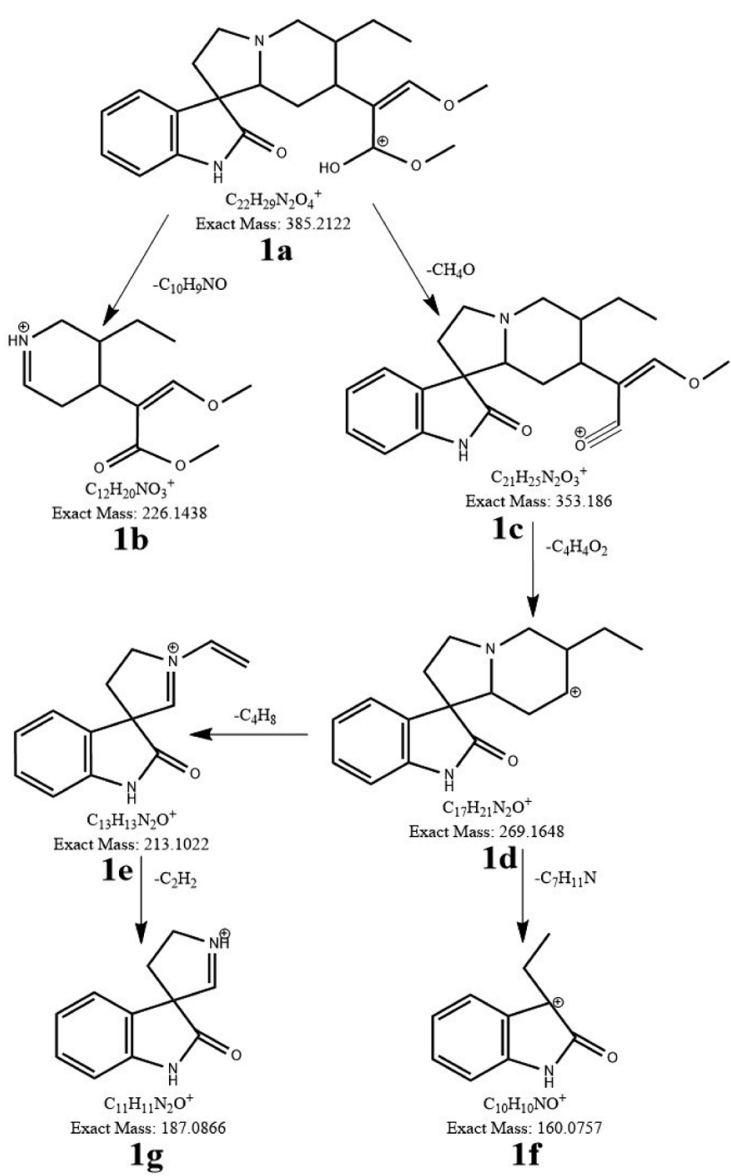

(2)

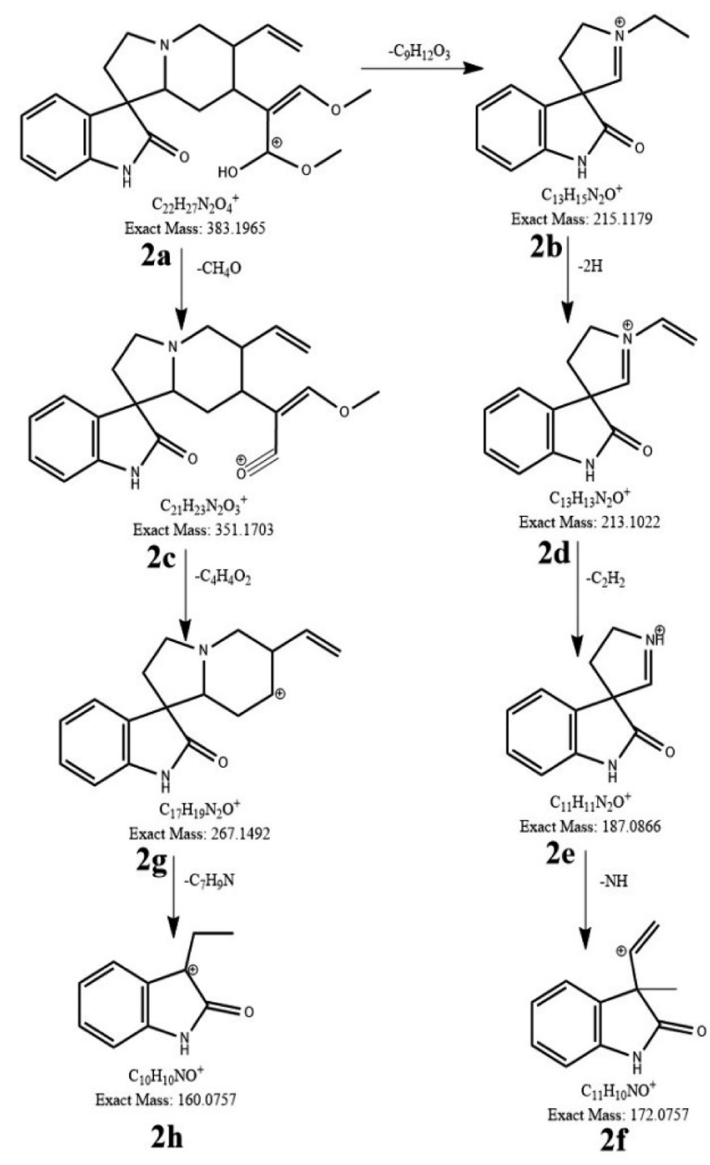

Fig. 4. (1) The Proposed Fragmentation Pathways of Rhynchophylline and Isorhynchophylline; (2) The Proposed Fragmentation Pathways of Corynoxeine and Isocorynoxeine

graphic conditions.

Recovery

Took the standard solutions with the concentrations of 3,5 and $7 \mu \mathrm{g} / \mathrm{mL}$ to analysis under corresponding chromatographic conditions.

\section{Results and Discussion}

The Determination of the Known Alkaloids in KHR98 We analyzed the accurate molecular weight provided by QTOF-MS and the retention time from chromatography, compared the information with the standard substances, and then detected rhynchophylline, isorhynchophylline, corynoxeine and isocorynoxeine in KHR98 (Fig. 1). The total ion chromatogram (TIC) chromatography was shown in Fig. 2.

MS/MS Fragment Pathways of Rhynchophylline and Isorhynchophylline

According to the accurate molecular weight and fragmentation information provided by HPLC-Q-TOF-MS, it was likely to deduce the possible fragmentation pathways of compounds.

In positive MS mode, some fragmentation information could be delineated on the basis of the mass fragmentation pathways and the intensities of the ion peaks. ${ }^{11)}$ As shown in Figs. 3(A) and 3(B), rhynchophylline and isorhynchophylline displayed an accurate quasi-molecular ion $[\mathrm{M}+\mathrm{H}]^{+}$at $m / z$ 385.2122, the molecular formula was $\mathrm{C}_{22} \mathrm{H}_{29} \mathrm{~N}_{2} \mathrm{O}_{4}^{+}$, and the characteristic fragmentation ions appeared at $\mathrm{m} / \mathrm{z} 226.14$,
$353.19,213.10,269.16,187.09$ and 160.08. The possible fragmentation pathways of rhynchophylline and isorhynchophylline could thus be inferred and the fragments observed further proved our prediction of those compositions (Fig. 4(1)). The loss of $\mathrm{C}_{10} \mathrm{H}_{9} \mathrm{NO}(159 \mathrm{Da})$ and methoxyl moiety (32 Da) unit from rhynchophylline and isorhynchophylline led to the emergence of the ion $\mathbf{1 b}$ at $\mathrm{m} / \mathrm{z} 226.14$ and the ion $\mathbf{1 c}$ at $\mathrm{m} / \mathrm{z} 353.19$. The fragment ion 1d $(\mathrm{m} / \mathrm{z} 269.16)$ could be explained by the elimination of $\mathrm{C}_{4} \mathrm{H}_{4} \mathrm{O}_{2}(84 \mathrm{Da})$ on 1c. Losing $\mathrm{C}_{4} \mathrm{H}_{8}(56 \mathrm{Da})$ and $\mathrm{C}_{7} \mathrm{H}_{11} \mathrm{~N}(109 \mathrm{Da}), \mathbf{1 d}$, as shown in its MS/MS spectrum, gave rise to the major fragment ions $\mathbf{1 f}(\mathrm{m} / \mathrm{z} 160.08)$ and $\mathbf{1 e}(\mathrm{m} / \mathrm{z}$ 213.10) which in turn generated ion $\mathbf{1 g}(\mathrm{m} / \mathrm{z}$ 187.09) with the removal of $\mathrm{C}_{2} \mathrm{H}_{2}(28 \mathrm{Da})$ segment from 1 e.

MS/MS Fragment Pathways of Corynoxeine and Isocorynoxeine

As shown in Figs. 3(C) and 3(D), the Q-TOF mass spectrometry of corynoxeine and isocorynoxeine saw the $[\mathrm{M}+\mathrm{H}]^{+}$ ion at $\mathrm{m} / \mathrm{z} 383.1965$, corresponding to the molecular formula $\mathrm{C}_{22} \mathrm{H}_{27} \mathrm{~N}_{2} \mathrm{O}_{4}^{+}$. A series of ions $(\mathrm{m} / \mathrm{z}$ 215.12, 351.17, 213.10, $267.15,187.09,160.08$ and 172.08) came out when corynoxeine and isocorynoxeine served as the parent ions. The possible fragmentation pathways of orynoxeine and isocorynoxeine were deduced and shown in Fig. 4(2). The appearance of the ion peaks at $\mathrm{m} / \mathrm{z} 215.12$ (2b) and $\mathrm{m} / \mathrm{z} 351.17$ (2c) could be attributed to the loss of $\mathrm{C}_{9} \mathrm{H}_{12} \mathrm{O}_{3}(168 \mathrm{Da})$ and methoxyl moiety (32 Da) unit from 2a. $2 d$ at $\mathrm{m} / z$ 213.10, which was created by 


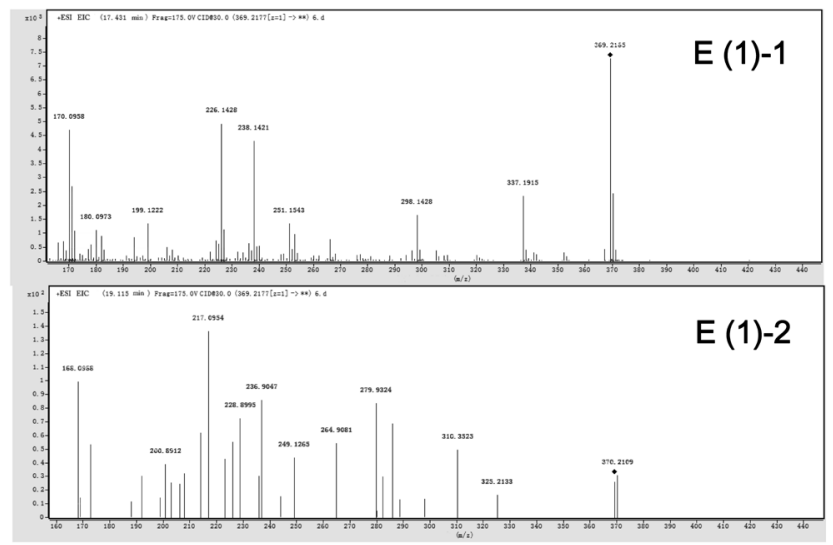

$E(2)$

$\mathrm{E}(3)$
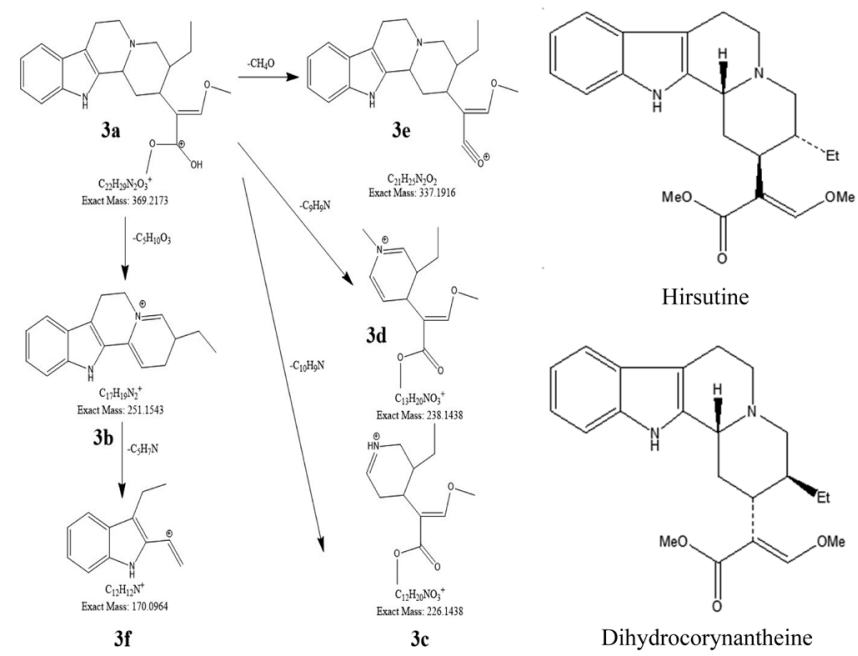

Dihydrocorynantheine

Fig. 5. $\mathrm{E}(1)-1$ MS/MS Spectra of the Unknown Alkaloids $\left(t_{\mathrm{R}}=\right.$ $17.431 \mathrm{~min}) ; \mathrm{E}(1)-2 \mathrm{MS} / \mathrm{MS}$ Spectra of the Unknown Alkaloids $\left(t_{\mathrm{R}}=\right.$ $19.115 \mathrm{~min}) ; \mathrm{E}(2)$ The Proposed Fragmentation Pathways of Alkaloids A1 and A2; E(3) Chemical Structures of Alkaloids A1 and A2 Identified in KHR98

2b after the elimination of two hydrogen atoms (2Da), could thus form ion $2 \mathbf{e}(\mathrm{m} / \mathrm{z} 187.09)$ after the removal of the chemical group $\mathrm{C}_{2} \mathrm{H}_{2}(26 \mathrm{Da})$. Then $2 \mathrm{f}(\mathrm{m} / \mathrm{z}$ 172.08) was produced with 2e losing NH (15Da). As for another pathway, $2 \mathbf{h}(\mathrm{m} / \mathrm{z}$ 160.08) was shaped after stripping $\mathrm{C}_{7} \mathrm{H}_{9} \mathrm{~N}$ group (107 Da) from $\mathbf{2 g}(\mathrm{m} / \mathrm{z} 267.15)$, which had the same structure as $\mathbf{2 c}$ after adding a chemical unit of $\mathrm{C}_{4} \mathrm{H}_{4} \mathrm{O}_{2}(84 \mathrm{Da})$.

The Identification of the Unknown Alkaloids in KHR98 Four main unknown alkaloids were identified in KHR98, their $[\mathrm{M}+\mathrm{H}]^{+}$ions were used as the precursor ions to optimize the collision energy and the MS/MS spectra were as well collected. Based on the fragmented ion information, the fragmentation regularity and relevant researches, the possible structure of the unknown components was deduced.

Structural Characterization and MS/MS Fragment Pathways of Alkaloids A1 and A2 Two unknown alkaloids (called alkaloids A1 and A2) were identified in KHR98, with their parent ion $[\mathrm{M}+\mathrm{H}]^{+}$being $\mathrm{m} / \mathrm{z} 369.2173$ and the retention time at 17.431 and $19.115 \mathrm{~min}$, respectively. The MS/MS spectra were shown in Figs. 5E(1)-1 and 5E(1)-2. Ions at $\mathrm{m} / \mathrm{z}$ $337.19,251.15,238.14,226.14$ and 170.10 could be used as characteristic fragmentation ions for alkaloids A1 and A2. According to the literature researches, alkaloids A1 and A2 may

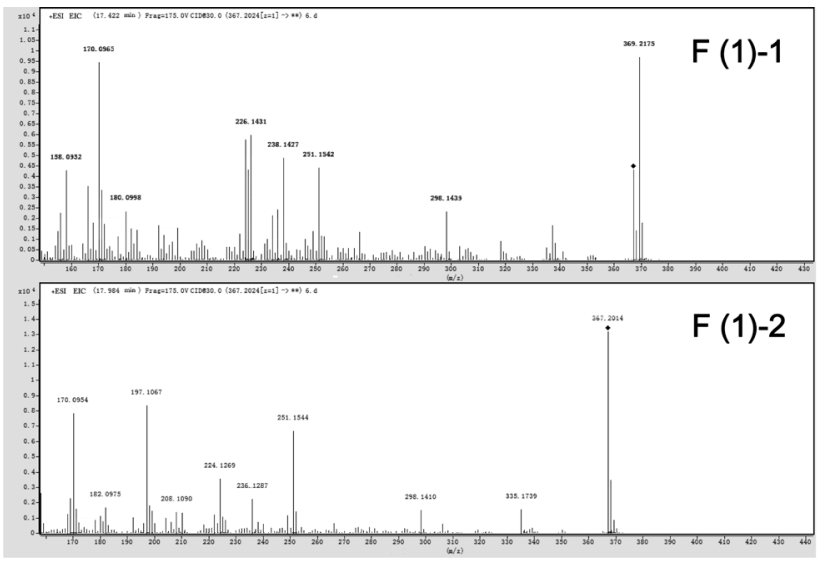

$F(2)$

$F(3)$
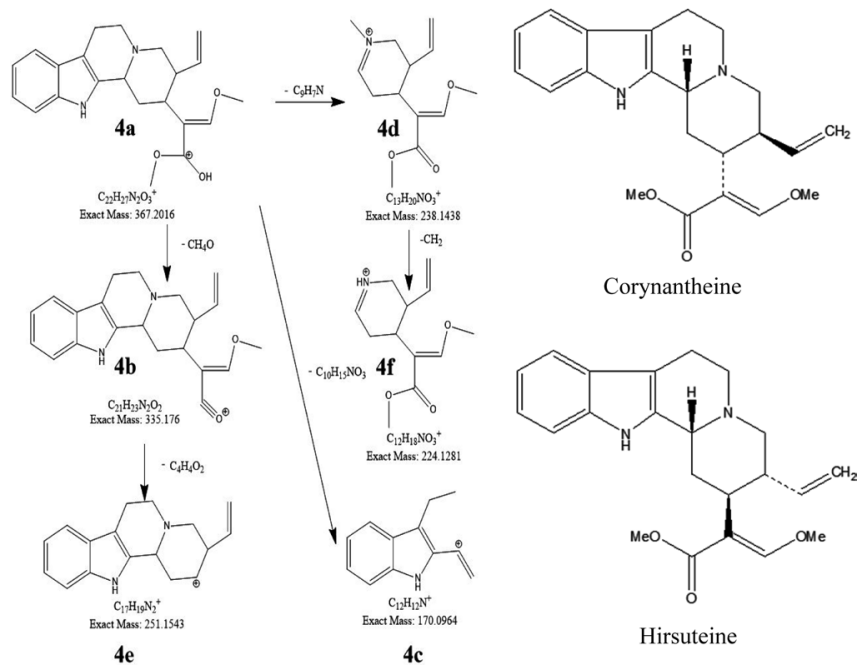

Corynantheine<smiles>C=C[C@H]1CN2CCc3c([nH]c4ccccc34)[C@@]2(C)C[C@@H]1/C(=C\OC)C(=O)O[Na]</smiles>

Hirsuteine

Fig. 5. F(1)-1 MS/MS Spectra of the Unknown Alkaloids $\left(t_{\mathrm{R}}=\right.$ $17.422 \mathrm{~min}) ; \mathrm{F}(1)-2 \mathrm{MS} / \mathrm{MS}$ Spectra of the Unknown Alkaloids $\left(t_{\mathrm{R}}=\right.$ $17.894 \mathrm{~min})$; F(2) The Proposed Fragmentation Pathways of Alkaloids B1 and B2; F(3) Chemical Structures of Alkaloids B1 and B2 Identified in KHR98

be hirsutine, dihydrocorynantheine or theirs isomers, whose structures were shown in Fig. $5 \mathrm{E}(3) .{ }^{15-18)}$ The possible fragment pathways were shown in Fig. $5 \mathrm{E}(2)$. In positive mode, the analyses of alkaloids $\mathrm{A} 1$ and $\mathrm{A} 2$ indicated the $[\mathrm{M}+\mathrm{H}]^{+}$ ion (3a) was at $\mathrm{m} / \mathrm{z} 369.2173$, corresponding to the molecular formula $\mathrm{C}_{22} \mathrm{H}_{29} \mathrm{~N}_{2} \mathrm{O}_{3}{ }^{+}$. Compounds $3 \mathbf{a}$ produced $\mathbf{3 b}, \mathbf{3 c}, \mathbf{3 d}$ and 3e, suggesting the loss of $\mathrm{C}_{5} \mathrm{H}_{10} \mathrm{O}_{3}(118 \mathrm{Da}), \mathrm{C}_{10} \mathrm{H}_{9} \mathrm{~N}(143 \mathrm{Da})$, $\mathrm{C}_{9} \mathrm{H}_{9} \mathrm{~N}(131 \mathrm{Da})$ and methoxyl moiety $(32 \mathrm{Da})$. The fragment ion $\mathbf{3 b}$ generated the ion $\mathbf{3 f}$ at $\mathrm{m} / \mathrm{z} 170.10$ due to the loss of $\mathrm{C}_{5} \mathrm{H}_{7} \mathrm{~N}(81 \mathrm{Da})$.

Structural Characterization and MS/MS Fragment Pathways of Alkaloids B1 and B2 Other two unknown alkaloids (called alkaloids B1 and B2) were identified in the KHR98, with their parent ion $[\mathrm{M}+\mathrm{H}]^{+}$being $\mathrm{m} / \mathrm{z} 367.2024$ and the retention time at 17.422 and $17.894 \mathrm{~min}$, respectively. The MS/MS spectra were shown in Figs. $5 \mathrm{~F}(1)-1$ and $5 \mathrm{~F}(1)-2$, in which a series of ions at $m / z 335.18,170.10,238.14,251.15$ and 224.13 arose from the alkaloids B1 and B2 as characteristic fragmentation ions. Based on literature data, alkaloids B1 and B2 were assigned as corynantheine, hirsuteine or theirs isomers, the structures of which were shown in Fig. $5 \mathrm{~F}(3),{ }^{15,16,19)}$ and the possible fragment pathways of which were 


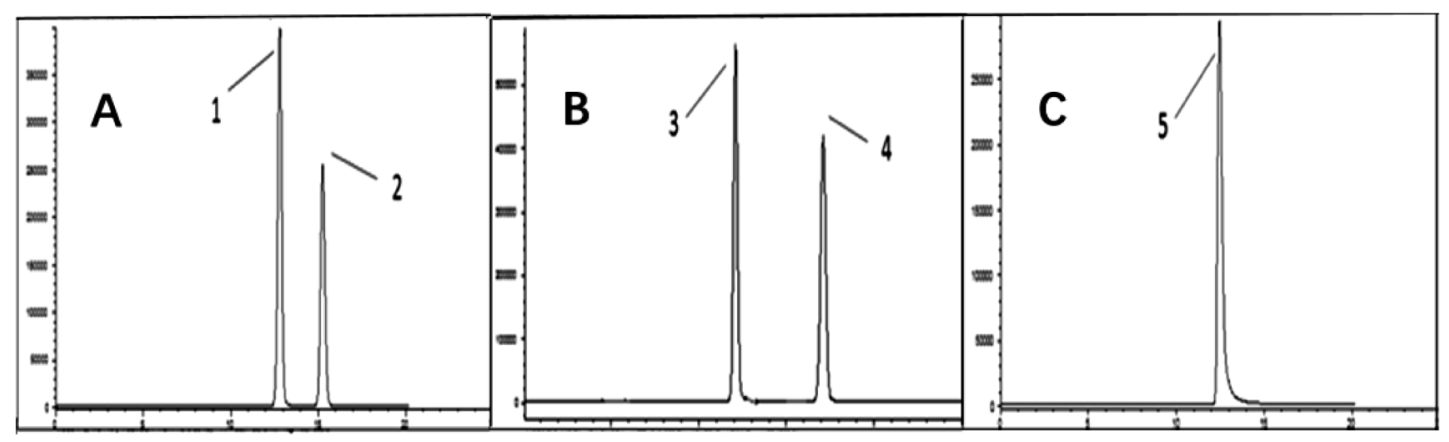

Fig. 6. EIC of Reference Solution (A. EIC=383.2, 1: Corynoxeine, 2: Isocorynoxeine; B. EIC=385.2, 3: Isorhynchophylline, 4: Rhynchophylline; C. $\mathrm{EIC}=369.2,5$ : Hirsutine)

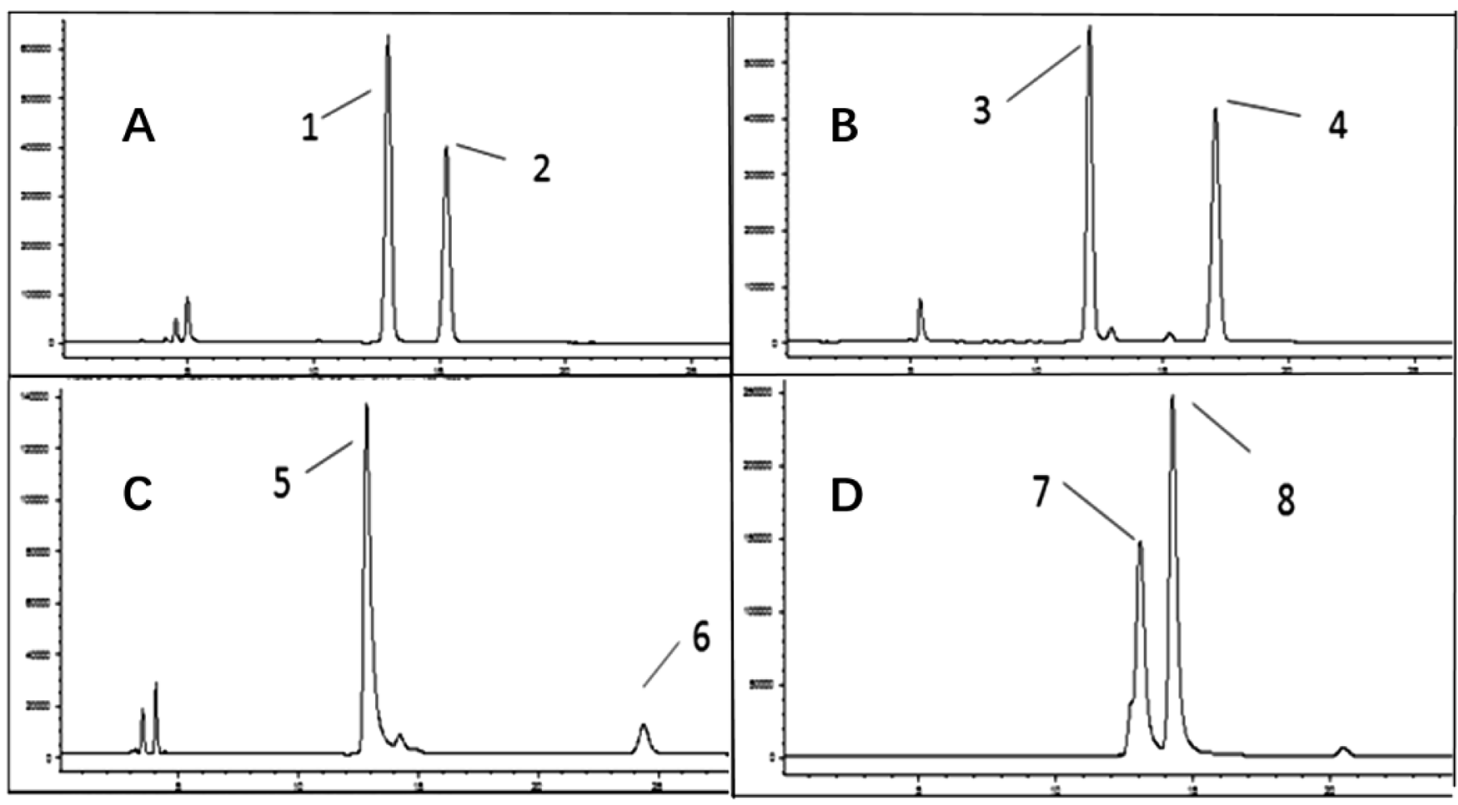

Fig. 7. EIC of Sample Solution (A. EIC=383.2, 1: Corynoxeine, 2: Isocorynoxeine; B. EIC=385.2, 3: Isorhynchophylline, 4: Rhynchophylline; C. $\mathrm{EIC}=369.2$, 5: Hirsutine, 6: Alkaloid A2; D. EIC=367.2, 7: Alkaloid B1, 8: Alkaloid B2)

Table 2. Linear Regression, LOD and LOQ of Five Investigated Compounds

\begin{tabular}{lccccc}
\hline \hline Alkaloid & Linearity range $(\mu \mathrm{g} / \mathrm{mL})$ & Regression equation $(n=5)$ & $r^{2}$ & LOD $(\mu \mathrm{g} / \mathrm{mL})$ & LOQ $(\mu \mathrm{g} / \mathrm{mL})$ \\
\hline Rhynchophylline & $1.16-11.6$ & $y=2576251 x+178847$ & 0.9994 & 0.04 & 0.22 \\
Isorhynchophylline & $1.09-10.9$ & $y=2461471 x+114735$ & 0.9993 & 0.03 & 0.18 \\
Corynoxeine & $1.3-13.0$ & $y=3636004 x+642168$ & 0.9991 & 0.08 & 0.07 \\
Isocorynoxeine & $1.2-12.0$ & $y=2705735 x+198143$ & 0.9996 & 0.45 \\
Hirsutine & $1.02-10.2$ & $y=2489374 x+209347$ & 0.9995 & 0.03 & 0.19 \\
\hline
\end{tabular}

Table 3. Inter-day and Intra-day of Five Investigated Compounds

\begin{tabular}{|c|c|c|c|c|c|c|}
\hline \multirow{2}{*}{ Alkaloid } & \multicolumn{3}{|c|}{ Intra-day precisions RSD (\%) } & \multicolumn{3}{|c|}{ Inter-day precisions RSD (\%) } \\
\hline & Low & Medium & High & Low & Medium & High \\
\hline Rhynchophylline & 1.46 & 1.39 & 1.52 & 1.74 & 1.82 & 1.99 \\
\hline Isorhynchophylline & 1.83 & 1.57 & 1.31 & 2.35 & 1.92 & 2.28 \\
\hline Corynoxeine & 1.36 & 1.44 & 1.57 & 1.84 & 1.63 & 2.17 \\
\hline Isocorynoxeine & 1.32 & 1.09 & 1.83 & 1.32 & 1.83 & 2.31 \\
\hline Hirsutine & 1.74 & 1.59 & 1.37 & 1.84 & 1.07 & 1.75 \\
\hline
\end{tabular}


Table 4. Recovery of Five Investigated Compounds

\begin{tabular}{lccr}
\hline \hline \multirow{3}{*}{ Alkaloid } & \multicolumn{3}{c}{ Recovery (\%) } \\
\cline { 2 - 4 } & Low & Medium & High \\
\hline Rhynchophylline & 98.39 & 100.86 & 100.36 \\
Isorhynchophylline & 97.57 & 99.28 & 99.11 \\
Corynoxeine & 98.45 & 99.39 & 101.12 \\
Isocorynoxeine & 98.35 & 101.02 & 100.62 \\
Hirsutine & 97.83 & 100.83 & 101.26 \\
\hline
\end{tabular}

Table 5. Contents of Eight Major Compounds in Three Batches of KHR98

\begin{tabular}{lrrr}
\hline \hline & \multicolumn{3}{c}{ Content $(\mathrm{mg} / \mathrm{g})$} \\
\cline { 2 - 4 } Batch number & \multicolumn{1}{c}{43} & \multicolumn{1}{c}{45} \\
\hline Rhynchophylline & 53.9 & 69.1 & 58.3 \\
Isorhynchophylline & 86.9 & 83.5 & 101.4 \\
Corynoxeine & 79.3 & 67.0 & 64.9 \\
Isocorynoxeine & 93.2 & 106.9 & 91.7 \\
Hirsutine & 31.0 & 54.0 & 45.0 \\
Dihydrocorynantheine & 3.0 & 5.0 & 6.0 \\
Corynantheine & 40.0 & 64.0 & 39.0 \\
Hirsuteine & 38.9 & 53.0 & 53.0 \\
Total content & 426.2 & 502.5 & 459.3 \\
\hline
\end{tabular}

shown in Fig. $5 \mathrm{~F}(2)$. Compound $\mathbf{4 a}$ produced ions $\mathbf{4 b}-\mathbf{4 d}$ in which $4 \mathbf{b}(\mathrm{m} / \mathrm{z} 335.18), 4 \mathbf{c}(\mathrm{m} / \mathrm{z} 170.10)$ and $4 \mathbf{d}(\mathrm{m} / \mathrm{z} 238.14)$ were assigned to the elimination of methoxyl moiety (32 Da), $\mathrm{C}_{10} \mathrm{H}_{15} \mathrm{NO}_{3}(197 \mathrm{Da})$, and $\mathrm{C}_{9} \mathrm{H}_{7} \mathrm{~N}(129 \mathrm{Da})$. Then $4 \mathbf{b}$ lost $\mathrm{C}_{4} \mathrm{H}_{4} \mathrm{O}_{2}$ (84Da) to generate ions $4 \mathbf{e}(\mathrm{m} / z$ 251.15). The ion $4 \mathbf{f}(\mathrm{m} / \mathrm{z}$ 224.13) arose from $4 d$ losing $\mathrm{CH}_{2}(14 \mathrm{Da})$.

\section{Method Validation of the HPLC-MS Analysis}

In this study, five marker compounds, namely corynoxeine, isocorynoxeine, rhynchophylline, isorhynchophylline and hirsutine, were simultaneously quantified by the developed quantitative method. SFDA guidelines were the foundation for establishing this analytical method. Extracted ion chromatography (EIC) mode with a four-section switch was employed for quantification. The corynoxeine and isocorynoxeine were at $\mathrm{m} / \mathrm{z}$ 383.2, rhynchophylline and sorhynchophyllineh were at $\mathrm{m} / \mathrm{z} 385.2$, hirsutine and dihydrocorynantheine were at $\mathrm{m} / \mathrm{z}$ 369.2, corynantheine and hirsuteine were at $m / z$ 367.2. The typical chromatograms for a standard solution and sample solution were shown in Figs. 6 and 7, which clearly indicated good separation could be achieved. Tables 2-4 demonstrated the results of method validation. Good linear relationships for all alkaloid were shown $\left(r^{2} \geq 0.9991\right)$ and the LODs and LOQs were discovered to be in the ranges from $0.03-0.08 \mu \mathrm{g} / \mathrm{mL}$ to $0.18-0.58 \mu \mathrm{g} / \mathrm{mL}$ (Table 2). For all five compounds, the Relative Standard Deviation (RSD) of intraday and interday precision were found to be within the scope of 1.09-1.83 and $1.07-2.35 \%$ (Table 3). The average recoveries were calculated and the observed alkaloids exhibited good recovery rates of 97.57-101.26\% (Table 4). The five alkaloids (corynoxeine, isocorynoxeine, rhynchophylline, isorhynchophylline and hirsutine) turned out to be stable in the sample solution in $12 \mathrm{~h}$ at indoor temperature, with RSD values at 1.96, 1.51, 2.05, 2.42 and $2.73 \%$.
Alkaloids consisted in KHR98 were investigated under the above chromatographic conditions and by the quantification method proposed. According to the qualitative results of the unknown compositions of KHR98 by HPLC-Q-TOF-MS, a new reference standard, hirsutine (alkaloid A1), was purchased. For those alkaloid components of existing standard (rhynchophylline, isorhynchophylline, corynoxeine, isocorynoxeine and hirsutine), the external standard method was used. For other alkaloids, dihydrocorynantheine (alkaloid A2), corynantheine (alkaloid B1), hirsuteine (alkaloid B2), Their reference standards were not easy to obtain, the content of each alkaloid component was calculated based on the similar structure of hirsutine. On the one hand, dihydrocorynantheine, corynantheine, hirsuteine and hirsutine have a similar structure, dihydrocorynantheine and hirsutine, corynantheine and hirsuteine are geometric isomers, hirsutine and dihydrocorynantheine have more than two hydrogen atoms than corynantheine and hirsuteine. On the other hand, these four substances have resemble fragmentation pattern, for instance, $\alpha$-cleavage, DRA-cleavage, i-cleavage and so on, and we determined the position of the peaks by the results of HPLCQ-TOF-MS. Eight compounds were quantified simultaneously and the results were displayed in Table 5.

\section{Conclusion}

To summarize, the possible fragmentation pathway of the four kinds of alkaloids components in KHR98, namely rhynchophylline, isorhynchophylline, corynoxeine and isocorynoxeine have been inferred with the proposed HPLC-QTOF-MS method. In addition, the main unknown components of KHR98 have been investigated, and the structures and possible fragmentation pathways have been founded and summarized. At last, eight marker compounds have been simultaneously quantified and their contents have been determined with the proposed HPLC-MS method. These pre-clinical data provide the first firm basis for the clinical researches, and our data can be used for New Drug Application and quality assessment of KHR98.

Conflict of Interest The authors declare no conflict of interest.

\section{References and Notes}

1) Xie S., Shi Y., Wang Y., Wu C., Liu W., Feng F., Xie N., J. Pharm. Biomed. Anal., 81-82, 56-64 (2013).

2) Geng C. A., Huang X. Y., Ma Y. B., Hou B., Li T. Z., Zhang X. M., Chen J. J., J. Nat. Prod., 80, 959-964 (2017).

3) Shi J. S., Yu J. X., Chen X. P., Xu R. X., Acta Pharmacol. Sin., 24, 97-101 (2003).

4) Jiang W. W., Su J., Wu X. D., He J., Peng L. Y., Cheng X., Zhao Q. S., Nat. Prod. Res., 29, 842-847 (2015).

5) Lee J. S., Kim J., Kim B. Y., Lee H. S., Ahn J. S., Chang Y. S., J. Nat. Prod., 63, 753-756 (2000).

6) Heitzman M. E., Neto C. C., Winiarz E., Vaisberg A. J., Hammond G. B., Phytochemistry, 66, 5-29 (2005).

7) Zhang Y. B., Yang W. Z., Yao C. L., Feng R. H., Yang M., Guo D. A., Wu W. Y., Fitoterapia, 96, 39-47 (2014).

8) Hui S., Yang Y., Mi Z., Zhu G. X., Qi A., Ji W., Zhu Z., Neuroscience, 337, 355-369 (2016)

9) Yuan D., Ma B., Wu C., Yang J., Zhang L., Liu S., Wu L., Kano Y., J. Nat. Prod., 71, 1271-1274 (2008).

10) Kong F., Ma Q., Huang S., Yang S., Fu L., Zhou L., Dai H., Yu Z., 
Zhao Y., Nat. Prod. Res., 31, 1403-1408 (2017).

11) Wang H. B., Qi W., Zhang L., Yuan D., Chem. Pharm. Bull., 62, 1100-1109 (2014)

12) Qu J., Gong T., Ma B., Zhang L., Kano Y., Yuan D., Chem. Pharm. Bull., 60, 23-30 (2012).

13) Montoro P., Carbone V., Quiroz Jde D., De Simone F., Pizza C., Phytochem. Anal., 15, 55-64 (2004).

14) State Food and Drug Administration Bureau of Traditional Chinese Medicine, "Natural Drug Registration Classification and Declaration of Information Requirements [EB/OL]": 〈http://www.sda.gov.
cn/WS01/CL0053/24529 9.html>, 2007.

15) Phillipson J. D., Supavita N., Phytochemistry, 22, 1809-1813 (1983).

16) Phillipson J. D., Hemingway S. R., Ridsdale C. E., Part V., Lloydia, 41, 503-570 (1978).

17) Laus G., Brössner D., Keplinger K., Phytochemistry, 45, 855-860 (1997)

18) Ponglux D., Supavita T., Verpoorte R., Phillipson D., J. Pharm. Pharmacol., 32 (S1), 2013-2016 (1980).

19) Laus G., Teppner H., Phyton, 36, 185-196 (1996). 